\title{
MEDICINE AS PRACTICED BY THE CHINESE
}

\author{
By William W. Cadbury, M.D., \\ University Medical School, Canton, China.
}

In the preparation of this article I have referred largely to a work entitled "Medicine et Pharmacie chez les Chinois et chez les Annamites" par le Dr. Jules Regnault, A. Challamei, Editeur, Paris, Rue Jacob I7. I have also included notes made from personal observations in Canton, China, and conversations with a Chinese scholar who had read some of the medical classics.

Medicine in China may be divided into two classes,- - the purely superstitious, which depends on charms and magic, and the art of medicine, as practiced by the Chinese physician. The former I shall dismiss with a few words. In the City of Canton may be found temples dedicated to the "Spirit of Medicine." In these the ignorant people, especially women, believe that the presiding deity will restore health upon the payment of small sums of money and the performance of certain rites. The Chinese physician, proper, is quite a different individual from the Taoist priest, although magic and astrology do play an important part in his armanentarium. Thus, for example, we read that as heaven has its orders of stars, so earth has its currents of water, and man his pulse. As heaven has twentyeight constellations called the three hundred and sixty-five orders, so earth has courses of water called lakes, springs, etc., and man has his courses in the pulse,--the three yang and the three yin. The practice of medicine is unlicensed and is usually hereditary, the skilled physician handing down his secrets to one of his sons. All the efforts of the students are directed to the pulse and the various phenomena revealed by its palpation. There are at least fifty-one variations in the pulse which may be detected and each one indicates some special condition in the body. For simple complaints home remedies and the formulas of old women are resorted to and only when grave symptoms develop is the doctor consulted. In case of warfare the Chinese soldiers attend to their own wounds.

The first authority on medicine in China was the Emperor Chen Song 2737 B.C. who classified about one hundred medicinal plants.

(124) 
In 2637 B.C. medical science, so far as it had advanced, was written up by another Emperor.

The Chinese distinguish three kinds of practice:-Internal medicine, external medicine and children's diseases.

The drugs and other medicaments are weighed out according to a decimal system as follows:

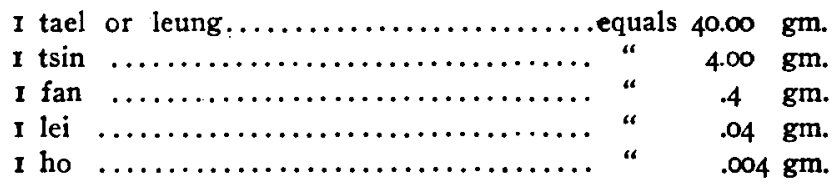

The study of human anatomy has been retarded by two factors, -respect for the dead and the lack of any co-operation or organization among the practicing physicians. The body is said to be divided into three parts: (I) The upper, or head; (2) the middle or chest; (3) the lower or abdomen and inferior extremities. Life depends on the equilibrium of the yang and the yin. It is but one manifestation of the universal life. The whole order of the universe results from the perfect equilibrium of these two factors. The yang is the warm principle, the actively flowing and is often symbolized by the sun. The yin is the moist principle, passively flowing and is symbolized by. shadow. The equilibrium of these two forces constitutes the health of man. If the yang, or active principle predominates there is excitation; if the yin, or passive principle predominates there is depression of the organism. Harmony between the yang and the yin is often represented by two dragons ready to devour one another. The action of these two principles depends on twelve organs: heart, liver, lungs, spleen, left kidney, brain, the large and small intestines, the stomach, gall bladder, urinary bladder and the right kidney. Each of these organs has a canal whereby it communicates with the others. Thus the liver, kidney and spleen are connected with the heart by special vessels; and the vas deferens arises from the kidney. Some of these communicating channels end in the hands and some in the feet. One of the vessels in the little finger is used to determine the nature of most infantile diseases. Six of these vessels carry the active principle yang, and six carry the passive principle yin. These two forces spread through the whole organism by means of the gases and the 
blood. The latter makes a complete circulation of the body about fifty times in twenty-four hours. In these fifty revolutions the blood passes twenty-five times through the male channels or those of the active principle and twenty-five times through the female channels, or those of the passive principle. The blood returns to its starting point every half hour approximately, instead of once in twenty-five seconds, according to the teaching of modern physiologists, having traversed a course of some fifty-four meters. The yang is of a subtle nature and resides in the abdomen and six viscera. It has a constant tendency to rise. The yin resides in the brain, the vertebral column and the five viscera and tends to descend.

The viscera of the body are classified under two groups:-the six viscera in which the yang resides and the five viscera in which the yin resides. The six viscera are: The gall bladder, stomach, small intestines, large intestines, bladder and the left kidney, with its three heat centers, the three lumbar sympathetic ganglia. The five viscera are: The heart, liver, lungs, spleen and right kidney. The diaphragm is placed beneath the heart and lungs, it covers over the intestines, spine and stomach. It is an impervious membrane. It covers over the foul gases, not allowing them to rise into the heart and lungs. The stomach, spleen and small intestines are the digestive organs. They prepare the blood which is received by the heart and set in motion by the lungs. The liver and the gall bladder filter out the various humors. The lungs expel the foul gases. The kidneys filter the blood, while coarser material is evacuated by the large intestines. Two substances are found circulating in the body, gases and blood. The former acts upon the latter as the wind upon the sea, the interaction of these two as they circulate in the vessels produces the pulse.

The pulse may be palpated at eleven different points, as follows: Radial, cubital, temporal, posterior auricular, pedal posterior tibial, external plantar, precordial and in three places over the aorta. Usually, however, the physician is satisfied with palpation of the pulse of the right and left wrist. With the right hand he feels the left pulse and with the left hand the right pulse. He applies three fingers, - the ring, middle and index over the pulse and the thumb underneath the wrist. Then he palpates the pulse with each finger successively. Under the ring finger the pulse of the right hand reveals the condition of the lung, middle of chest and the large 
intestines, while in the left hand the ring finger determines the state of the heart and small intestines. The pulse under the middle finger corresponds on the right to the condition of the stomach and spleen, on the left to the state of the liver and gall bladder. The index finger placed over the pulse of the right radial shows the condition of the bladder and lower portion of body, over the left radial it reveals the state of the kidneys and ureters. For each of these six pulses the physician must practice weak, moderate and strong pressure, to determine whether the pulse be superficial, moderate or deep. This must be done during nine complete inspirations. If the pulse be rapid the yang principle is predominant, if slow, the yin is predominant. There are twenty-four main varieties of pulse and there are twenty-seven which prognosticate death. The Chinese physician must be trained to palpate the pulse so skillfully that by this single means the nature of diseases and even the month of gestation in a pregnant woman may be determined. Ten or more minutes must be spent in the palpation of the pulses.

Sometimes a Chinese physician will consider other factors. For example it is said that by examination of the tongue thirty-six symptoms may be diagnosed according as the tongue is white, yellow, blue, red or black, and depending on the extent of the coating. From the general appearance of the face and nose the state of the lungs may be discovered. Examination of the eyes, orbits, and eyebrows shows the condition of the liver. The cheeks and tongue vary with the state of the heart, the end of the nose with the stomach. The ears suggest conditions of the kidneys; the mouth and lips the state of the spleen and stomach. The color and figure of the patient also count in a diagnosis. Each organ has its appropriate color. Red corresponds to the heart, white to the lungs, black to the kidneys and bladder, yellow to the stomach and spleen and blue to the liver and gall bladder. Organs also have their own peculiar times and seasons. Thus the heart has red as its color, fire as its element, summer as its season and noon as its hour. It is more likely to be inflamed at noon during the summer season. The elements of nature are supposed to be complicating factors in disease. They are arranged in pairs of opposites thus: active and passive, weak and strong, water and fire, cold and heat.

Auscultation and percussion are wholly unknown as diagnostic aids to the Chinese physician. Entire reliance is placed on palpation 
of the pulse and the general facies of the patient, in making a diagnosis. Questions may be asked but only to suggest the remedy required. Often a prescription is given because of the resemblance of the drug to the organ affected. Thus for renal diseases, haricot or kidney beans are given. Minerals are administered as salts. Plants are used in the form of roots, stems, leaves, flowers and dried fruits. The bones of a tiger are frequently ground up and given to a debilitated person. The grasshopper is dried and used as a medicine and the shells of the cicada are collected from the bark of trees and mixed with other ingredients. Tinctures and extracts are prepared from rice wine. Pills are often made with a thick shell of paraffine which is broken off and the contents chewed up. Various forms of plasters and blisters may be applied to the skin. The actual cautery is often used as a revulsive.

The use of the acupuncture needle seems to be seldom resorted to in the neighborhood of Canton. The theory on which it is based is that if one punctures the vessels connecting different organs the disease will be aborted. Three hundred and eighty-eight points suitable for acupuncture are described. Diseases of the liver and the eyes, which are sympathetic organs, are cured by giving pork's liver. In Kwongtung province human blood is considered an excellent remedy and at executions people may be seen collecting the blood in little vials. It is then cooked and eaten.

Diseases are said to be produced by internal and external agents. Among the external influences are: (I) Wind, which causes headache or apoplexy, dizziness, chapping of face, diseases of the eye, ear, nose, tongue, teeth, etc. (2) Cold may cause cough, cholera, heart pains, rheumatism and abdominal pains. (3) Heat causes chills and diarrhœa. (4) From dampness develops constipation, distention of abdomen, watery diarrhœa, gonorrhea, nausea, pain in kidneys, jaundice, anasarca, pain in small intestines, and pain in feet. (5) From dryness comes thirst, and constipation. (6) Fire causes pain in the sides, diabetes, etc. The diseases of internal origin are classified as disorders of the gases, blood, sputum and depressed spirits.

In the past few years there have been established two charitable institutions in Canton for the treatment of the sick, according to native methods of practice. No surgery is practiced. At one of these so-called hospitals I was informed that bullets were removed 
by placing a kind of plaster at the opening of the wound. The ingredients of the plaster have a remarkable magnetic power over the imbedded bullet and gradually draw it out through the same opening by which it entered. My informant had never seen this line of treatment actually carried out, however.

There is a great desire on the part of many Chinese young men to learn the science of western medicine, and in the next few years there will be a demand for thousands of Chinese trained as scientific physicians. 